Check for updates

Cite this: J. Mater. Chem. B, 2020 8,5330

Received 17th December 2019 Accepted 15th May 2020

DOI: $10.1039 / c 9 t b 02868 d$

rsc.li/materials-b

\section{Size-controlled clustering of iron oxide nanoparticles within fluorescent nanogels using LCST-driven self-assembly $\dagger$}

\author{
Turgay Yildirim, ${ }^{a}$ Maria Pervez, (D) ${ }^{a}$ Bo $\mathrm{Li}^{\mathrm{b}}$ and Rachel K. O’Reilly (D) *a
}

\begin{abstract}
Size-controlled clustering of iron oxide nanoparticles (IONPS) within the fluorescent polymer nanogels was achieved using the lower critical solution temperature (LCST) driven self-assembly and cross-linking of grafted polymer on the IONPs. The grafted polymer was comprised of oligoethyleneglycol methacrylate (OEGMA) and a novel dichloromaleimide functional methacrylate monomer as building blocks. As a result of the temperature responsive behavior of OEGMA, polymer grafted IONPs clustered to form larger nano-sized aggregates when heated above the LCST of the polymer. When these nanosized aggregates were cross-linked using an amine-dichloromaleimide reaction, well-defined fluorescent hybrid nanogels could be fabricated. Moreover, the size of these hybrid nanogels was effectively controlled by varying the initial concentration of the polymer grafted IONPs in water.
\end{abstract}

Iron oxide nanoparticles (IONPs) have been under intensive investigation for a variety of biomedical applications, such as magnetic resonance imaging (MRI), ${ }^{1}$ hyperthermia therapy, ${ }^{2}$ drug delivery, ${ }^{3}$ immunoassay ${ }^{4}$ as well as cell separation ${ }^{5}$ mainly due to their responsiveness to magnetic fields. When the size of IONPs is sufficiently small (around 10-30 nm), they exhibit superparamagnetic behavior, meaning that they rapidly respond to an applied external magnetic field similar to a paramagnet yet exhibit negligible residual magnetism when the external magnetic field is removed. ${ }^{6}$ This behavior avoids strong magnetic interactions between the individual IONPs and prevents/inhibits the agglomeration of IONPs in the absence of an applied magnetic field, which makes superparamagnetic IONPs very attractive for biomedical applications. However, the small size of the superparamagnetic IONPs results in a low magnetization per particle, limiting the manipulation of IONP movement using an external magnetic field. ${ }^{7}$ To overcome this limitation, increasing attention has been drawn to polymer assisted solution self-assembly of IONPs enabling access to IONPs embedded in colloidal polymer particles with a controlled size and morphology. ${ }^{8-13}$ The vast majority of these systems rely on block copolymer assisted solution self-assembly. ${ }^{14}$ As a seminal example, Taton and coworkers formulated polymer micelles loaded with IONPs using the simultaneous self-assembly of

\footnotetext{
${ }^{a}$ School of Chemistry, University of Birmingham, Edgbaston, Birmingham, B15 2TT, UK. E-mail: R.OReilly@bham.ac.uk

${ }^{b}$ Department of Chemistry, University of Warwick, Gibbet Hill Road, Coventry, CV4 $7 A L, U K$

$\dagger$ Electronic supplementary information (ESI) available. See DOI: 10.1039/c9tb02868d
}

amphiphilic copolymers and IONPs. ${ }^{15}$ Recently, Khashab and co-workers fabricated nanosized polymer vesicles having tunable layers of densely packed superparamagnetic IONPs using cooperative self-assembly of polymer tethered IONPs and a free block copolymer. ${ }^{16}$

Crosslinking of thermoresponsive amphiphilic random copolymers above their lower critical solution temperature (LCST) provides a facile approach to form stable nanogels in aqueous solutions. As a pioneering example, Thayumanavan and coworkers achieved efficient nanogel synthesis by heating a random copolymer, that contain cross-linkable disulfide units and thermoresponsive oligoethyleneglycol moieties as side-chain functionalities, above its LCST temperature, followed by in situ disulfide crosslinking mediated by the addition of dithiothreitol. ${ }^{17,18}$ Additionally, Sanyal and coworkers demonstrated that a poly(ethylene glycol)methacrylate-based, maleimide functional random copolymer can yield nanogels through cross-linking of copolymer with a dithiol-based cross-linker above the LCST temperature of the polymer in water. ${ }^{19}$ However, to the best of our knowledge, clustering of IONPs into polymer hydrogels using LCST driven self-assembly has never been reported in the open literature so far.

Herein, we report a novel approach for the size-controlled clustering of IONPs into polymer nanogels harnessing the thermoresponsive behavior of an amphiphilic random copolymer tethered on IONPs (Scheme 1). To synthesize IONP encapsulated nanogels, a statistical copolymer of poly(ethylene glycol) monomethyl ether methacrylate $\left(\mathrm{OEGMA}_{300}, M_{\mathrm{n}}=300 \mathrm{~g} \mathrm{~mol}^{-1}\right)$ and a dichloromaleimide (DCM) functional monomer (DCMMA) was synthesized by reversible addition-fragmentation chain transfer 
A)

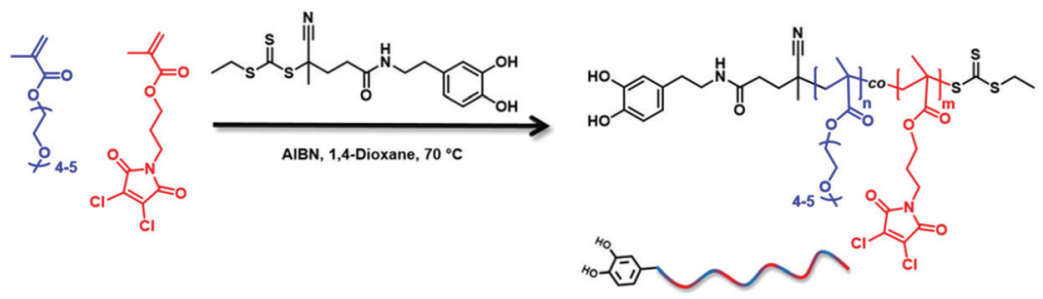

B)
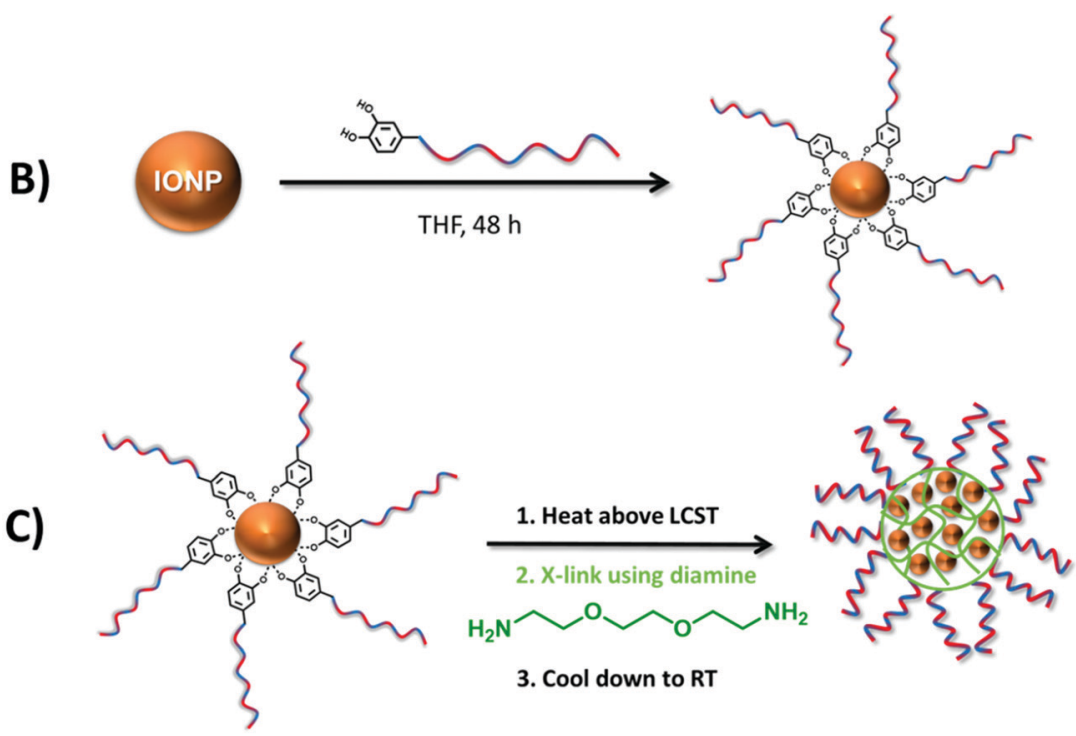

3. Cool down to RT

Scheme 1 Schematic representation of the (A) RAFT copolymerization of OEGMA 300 with DCMMA using the dopamine functional CTA, (B) grafting of the synthesized polymer on the iron oxide nanoparticles (C) clustering of IONPs using LCST driven self-assembly.

(RAFT) polymerization using a dopamine functional chain transfer agent (CTA) (Scheme 1A) and the statistical copolymer was tethered on IONPs (Scheme 1B). OEGMA based hydrophilic polymers are known for their biocompatibility, stealth effect as well as exhibiting LCST behavior with a tunable cloud point temperature $\left(T_{\mathrm{CP}}\right)$ between 20 and $90{ }^{\circ} \mathrm{C} \cdot{ }^{20,21}$ Additionally, surface coating of IONPs using OEGMA based polymers provides an effective method for the stabilization of IONPs in water, thereby avoiding agglomeration. ${ }^{22}$ Therefore, we used $\mathrm{OEGMA}_{300}$ as the main monomer in the polymer chains, which can stabilize the IONPs in water at room temperature but forms larger nanosized aggregates when heated above its LCST. Fast and efficient crosslinking of the nanosized aggregates is crucial in order to obtain stable nanogels with a monomodal size distribution. It is known that dibromomaleimide (DBM) functional polymers can be modified with primary amine or thiol bearing small molecules in water through single substitution under mild conditions with high efficiency. ${ }^{22,23}$ Previous work within our group has shown that DCM is even more reactive than DBM. ${ }^{24}$ Moreover, although DCM and DBM are not fluorescent, the substitution reaction of DBM and DCM with amine or thiol functional molecules yields fluorescent maleimide derivatives. ${ }^{23,25,26}$ This could provide a facile approach for the synthesis of fluorescent nanogels without any need of post-nanoparticle functionalization with a fluorescent dye. As a consequence, for the crosslinking of the nanogels we chose to use DCM diamine chemistry (Scheme 1C). The findings on the LCST-driven self-assembly behavior of the statistical copolymer as well as the copolymer tethered IONPs are disclosed herein.

First, a novel dichloromaleimide functional monomer, 3-(3,4dichloro-2,5-dioxo-2,5-dihydro-1 $H$-pyrrol-1-yl)propyl methacrylate (DCMMA), was synthesized as demonstrated in Scheme S1 (ESI $\dagger$ ). ${ }^{1} \mathrm{H}$ and ${ }^{13} \mathrm{C}$ NMR spectroscopic analysis proved the successful synthesis of the desired monomer (Fig. S1 to S4, ESI $\dagger$ ). Then, a dopamine functional chain transfer agent (CTA) was synthesized as depicted in Scheme S2 (ESI $\dagger$ ). Characterization by ${ }^{1} \mathrm{H}$ and ${ }^{13} \mathrm{C}$ NMR spectroscopy confirmed the desired structure of the CTA (Fig. S5 to S10, ESI†). A statistical copolymer of OEGMA 300 and DCMMA was synthesized using the dopamine functional CTA by utilizing the RAFT polymerization process (Scheme 1A). In order to obtain a water soluble polymer at room temperature, the initial monomer feed ratio ([OEGMA $\left.{ }_{300}\right] /[$ DCMMA]) was set to 4 (Table S1, ESI $\dagger$ ). A dopamine functional CTA was used in order to attach the synthesized polymer onto IONPs. ${ }^{27}$ Polymerization was carried out in dioxane at $70{ }^{\circ} \mathrm{C}$ with a total monomer concentration of $0.4 \mathrm{~mol} \mathrm{~L}^{-1}$. The monomer conversions for OEGMA $_{300}$ and DCMMA were determined as $62 \%$ and $68 \%$, respectively, by ${ }^{1} \mathrm{H}$ NMR using 1,3,5-trioxane as internal standard. The theoretical molar mass $\left(M_{\mathrm{n}, \text { theo. }}\right)$ of the copolymer was calculated as $19250 \mathrm{~g} \mathrm{~mol}^{-1}$ (see the Formula (S1) in the ESI $\dagger$ ). ${ }^{1} \mathrm{H}$ NMR spectroscopy analysis of the isolated copolymer clearly revealed the presence of the aromatic dopamine protons of $\alpha$-RAFT end groups ( $\delta=6.73,6.65$, and $6.51 \mathrm{ppm}$ ), and the characteristic signals that are derived from OEGMA $_{300}(\delta=4.0,3.59$ and $3.31 \mathrm{ppm})$ and 
DCMMA $(\delta=3.90 \mathrm{ppm})$ (Fig. S11, ESI $\dagger$ ). This enables the estimation of the degree of polymerization (DP) for OEGMA 300 as 52 and for DCMMA as 14 (see the Formulas (S2) and (S3) in the ESI $\dagger$ ) by comparing the integral values of the methine proton peak of the OEGMA $_{300}$ side chains at $\delta=4.0 \mathrm{ppm}$ (peak " 2 " in Fig. S11, ESI $\dagger$ ), the integral value of the methine proton peak of the DCMMA side chains at $\delta=3.90$ ppm (peak " 3 " in Fig. S11, ESI $\dagger$ ) and the integral value of aromatic dopamine protons of $\alpha$-RAFT end groups ( $\delta=6.73,6.65$, and $6.51 \mathrm{ppm})$ (peak " 1 ” in Fig. S11, ESI $\dagger$ ). The according number average molar mass $\left(M_{\mathrm{n}}\right)$ value $\left(20100 \mathrm{~g} \mathrm{~mol}^{-1}\right.$ ) calculated by ${ }^{1} \mathrm{H}$ NMR (see the Formula (S4) in the ESI $\dagger$ ) is in good agreement with the theoretical value. SEC analysis revealed a molar mass value of $13100 \mathrm{~g} \mathrm{~mol}^{-1}$ with a monomodal trace and a relatively low dispersity $\left(D_{M}=1.35\right)$ (Fig. S12, ESI $\dagger$ ). The underestimated molar mass by SEC was attributed to the PS calibration in the SEC system, resulting in a relative molar mass value.

The thermoresponsive behavior of the statistical copolymer in aqueous media was investigated using turbidimetry measurements with a heating and cooling rate of $1{ }^{\circ} \mathrm{C} \mathrm{min}^{-1}$. Two heating/cooling cycles were conducted in a temperature range between 20 and $80{ }^{\circ} \mathrm{C}$. Turbidimetry measurements revealed that the polymer was completely soluble in water at low temperatures, but an increase of temperature above $37{ }^{\circ} \mathrm{C}$ resulted in a drastic decrease of the transmittance (Fig. 1). The $T_{\mathrm{CP}}$ of the polymer was calculated to be $40{ }^{\circ} \mathrm{C}$ (the temperature where the transmittance decreased to $50 \%$ in the second heating run). According to literature, homopolymers of OEGMA ${ }_{300}$ exhibit $T_{\mathrm{CP}}$ above $60{ }^{\circ} \mathrm{C}$ with a strong dependence on the molar mass. ${ }^{20}$ The lower $T_{\mathrm{CP}}$ of the synthesized statistical copolymer compared with homopolymers of OEGMA 300 was attributed to the hydrophobic DCMMA units within the polymer. ${ }^{28}$ The temperature responsive behavior of the polymer in water was also investigated by performing DLS measurements at different temperatures. In agreement with the turbidimetry measurements, at low temperatures, DLS revealed hydrodynamic diameters below $10 \mathrm{~nm}$ (Fig. 1). However, DLS revealed a drastic increase in the hydrodynamic diameter of the polymer at $45{ }^{\circ} \mathrm{C}$. After cooling, the original size distribution was obtained, which indicates the reversibility of the temperature responsive behavior of the polymer (Fig. 1).

Prior to tethering the synthesized polymer on iron oxide nanoparticles, it was important to prove that the synthesized polymer can form nanogels by heating above its LCST and cross-linking via an amine-dichloromaleimide reaction. To this end, the polymer was dissolved in water $\left(2 \mathrm{mg} \mathrm{mL}^{-1}\right)$ and heated at $70{ }^{\circ} \mathrm{C}$ for $1.5 \mathrm{~min}$. After $1.5 \mathrm{~min}$, a calculated amount of 2,2'-(ethylenedioxy)bis(ethylamine) aqueous solution as crosslinker was added into the polymer solution and the solution was stirred at $70{ }^{\circ} \mathrm{C}$ for $20 \mathrm{~min}$, followed by cooling down to room temperature. The molar ratio of the dichloromaleimide and the cross-linker was set to $1: 1$. In order to confirm the reproducibility of nanogel synthesis, this procedure was repeated three times. A schematic illustration of the polymer nanogel preparation is shown in Scheme S3 (ESI $\dagger$ ). The colorless and clear aqueous polymer solution at room temperature became turbid when heated at $70{ }^{\circ} \mathrm{C}$. After the addition of the cross-linker, the polymer solution became yellow and it maintained its yellow color as well as its turbidity when cooled to room temperature. The obtained assemblies were characterized using DLS without any filtration. DLS revealed that all batches have very similar intensity average diameters $\left(D_{\mathrm{av}}=200 \pm 5 \mathrm{~nm}\right)$ with monomodal size distributions and low polydispersity index (PD) values (lower than 0.08) (Fig. S13 and Table S2, ESI $\dagger$ ). These results prove the reproducibility of the nanogel synthesis. Then, nanogels synthesized from three different batches were combined and characterized by DLS and TEM. DLS revealed a $D_{\mathrm{av}}$ of $197 \pm 2 \mathrm{~nm}$ with a PD value of $0.03 \pm$ 0.02 and zeta potential measurements showed a negative zeta potential value $(-21 \pm 2 \mathrm{mV})$ (Fig. 2). TEM analysis revealed
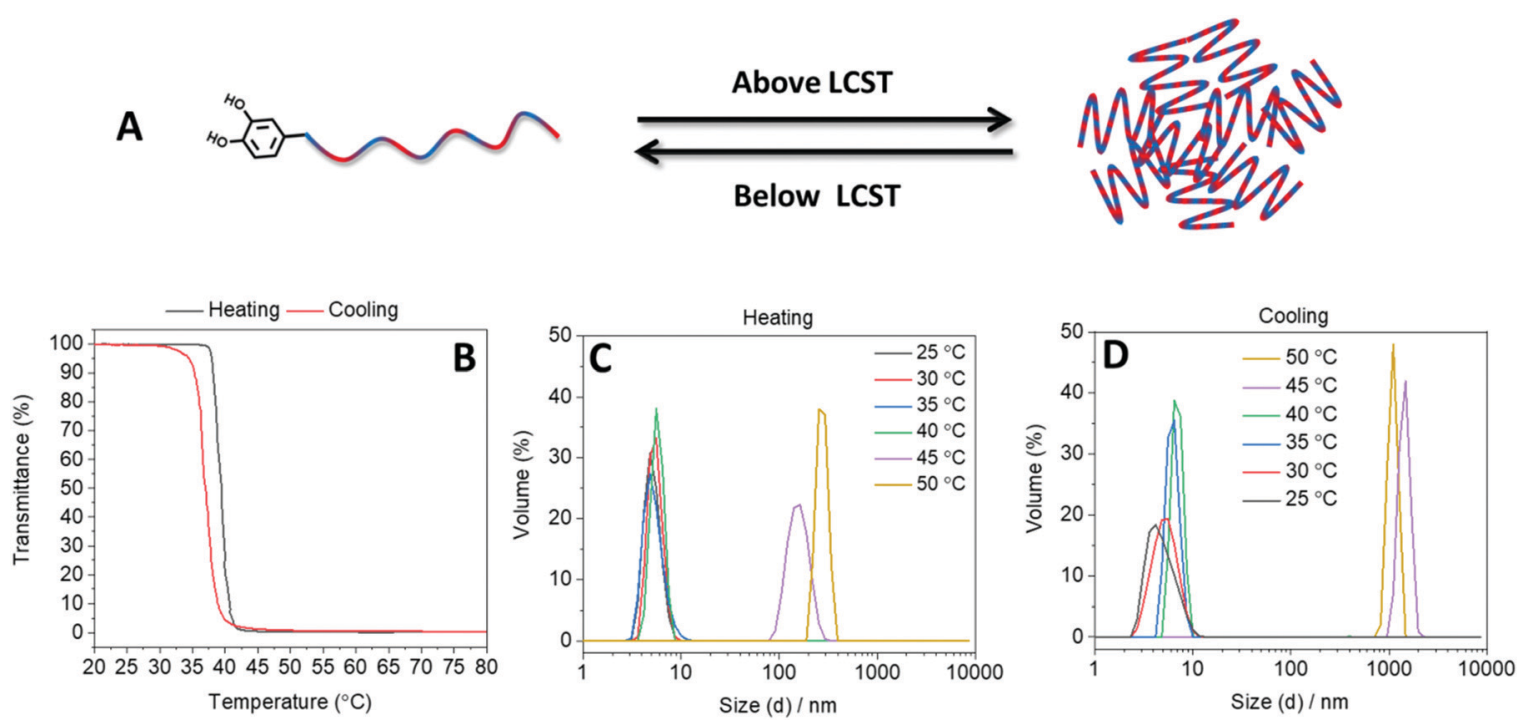

Fig. 1 (A) Schematic depicting of reversible LCST-driven self-assembly behavior of the polymer. (B) Variable temperature turbidimetry analysis of the polymer at $2 \mathrm{mg} \mathrm{mL}^{-1}$ in water (heating and cooling rate $=1{ }^{\circ} \mathrm{C} \mathrm{min}{ }^{-1}$ ). DLS size distributions of the polymer in water at $2 \mathrm{mg} \mathrm{mL}^{-1}$ as a function of the temperature (C; heating, D; cooling). 

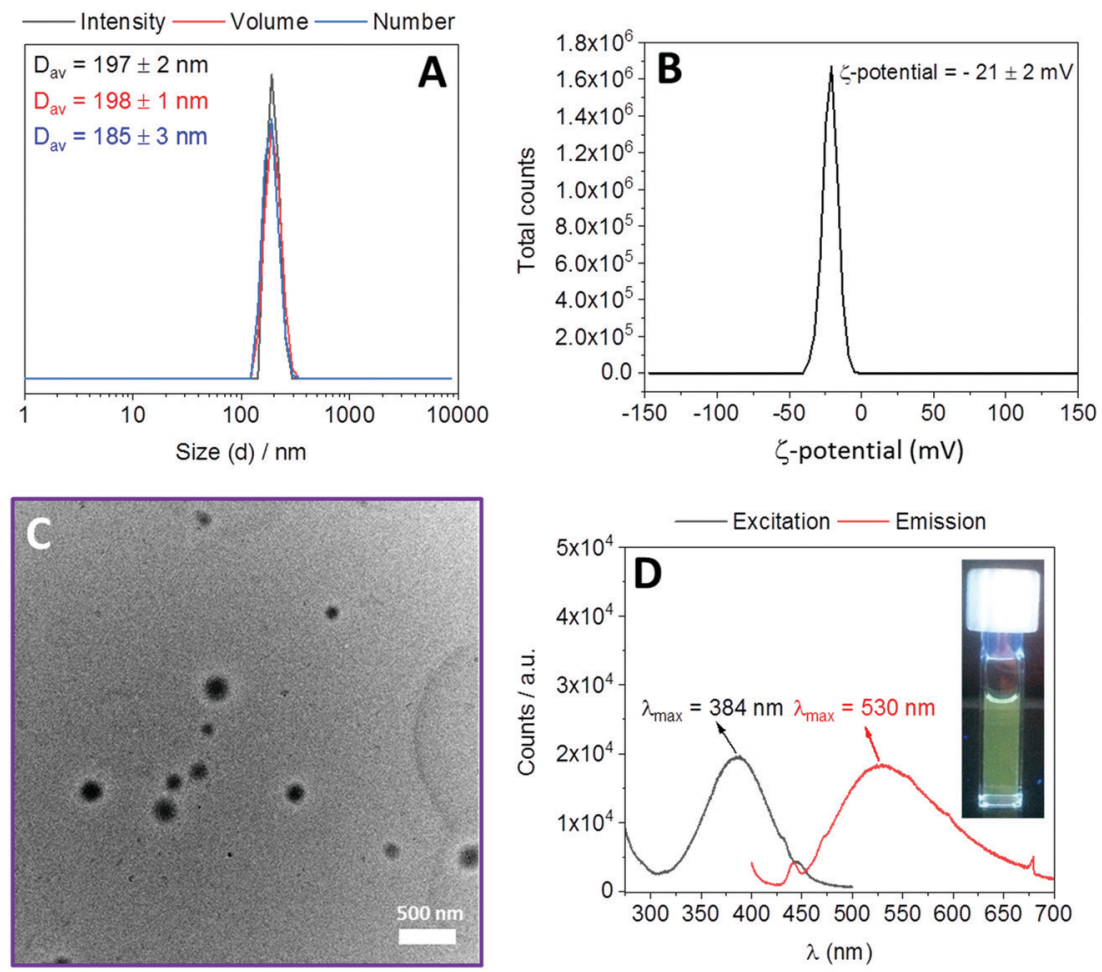

Fig. 2 (A) DLS size distributions, (B) $\zeta$-potential distribution of nanogels, (C) TEM images of the nanogels synthesized with an initial polymer concentration of $2 \mathrm{mg} \mathrm{mL}^{-1}$ (sample was stained using $1 \mathrm{wt} \%$ uranyl acetate (UA) solution) and (D) excitation and emission spectra of nanogels in water $\left(c=0.165 \mathrm{mg} \mathrm{mL}^{-1}\right.$ ), (inset: photograph of solution of nanogels under UV light at $365 \mathrm{~nm}$ ).

well-defined spherical morphologies to the synthesized nanogels with diameters (from 120 to $170 \mathrm{~nm}$ ) slightly smaller than diameters obtained in DLS (Fig. 2). The formation of the nanogels was attributed to the reaction of diamine cross-linker with the dichloromaleimide functionalities within the aggregated polymer. The appearance of a strong absorption band at $\lambda_{\max }=383 \mathrm{~nm}$ in the UV-vis spectrum of the nanogels, which is the characteristic signal of substituted maleimides ${ }^{24}$ proves this assumption (Fig. S14, ESI $\dagger$ ). Additionally, when excited at $384 \mathrm{~nm}$, nanogels showed a fluorescence emission with $\lambda_{\max }=530 \mathrm{~nm}$ (Fig. 2). To investigate the effect of initial polymer concentration in aqueous media on the size of the nanogels, the same experiments were conducted at two additional concentrations $\left(1\right.$ and $\left.4 \mathrm{mg} \mathrm{mL}^{-1}\right)$. DLS measurements showed that $1 \mathrm{mg} \mathrm{mL}{ }^{-1}$ yielded smaller nanogels $\left(D_{\mathrm{av}}=178 \pm 2 \mathrm{~nm}\right)$ and $4 \mathrm{mg} \mathrm{mL}{ }^{-1}$ yielded larger nanogels $\left(D_{\mathrm{av}}=334 \pm 6 \mathrm{~nm}\right)$ compared to $2 \mathrm{mg} \mathrm{mL}^{-1}\left(D_{\mathrm{av}}=197 \pm\right.$ $2 \mathrm{~nm}$ ) (Fig. S15, ESI $\dagger$ ). These results indicate that an increase of the polymer concentration results in an increase of the size of the synthesized nanogels. This is in agreement with previously published results. ${ }^{17-19}$

Oleic acid-stabilized IONPs were synthesized using a thermal decomposition method as reported previously. ${ }^{29}$ DLS measurements in hexane revealed monomodal size distributions with number average hydrodynamic diameter $\left(D_{\mathrm{n}}\right)$ of $18 \pm 3 \mathrm{~nm}$ (Fig. S16, ESI $\dagger$ ). In agreement with DLS, TEM showed spherical IONPs with diameters around 10 to $20 \mathrm{~nm}$ (Fig. S16, ESI $\dagger$ ). The iron oxide content of the IONPs were investigated by thermogravimetric analysis (TGA). The weight loss was determined to be $39 \%$ (Fig. S17, ESI $†$ ).
Dopamine terminated polymer was grafted onto the surface of IONPs using a ligand exchange reaction based on the chelation of dopamine with the surface of IONPs (Scheme 1B), yielding P-IONp. ${ }^{27}$ TGA measurements revealed that P-IONp have $27 \%$ iron oxide content (Fig. S18, ESI $\dagger$ ). TEM images showed no change in the diameter of the IONPs after polymer grafting (Fig. S19, ESI $\dagger$ ). DLS measurements in water revealed that P-IONp had a slightly larger number average hydrodynamic diameter $\left(D_{\mathrm{n}}=32 \pm 6 \mathrm{~nm}\right)$, than polymer free IONPs $\left(D_{\mathrm{n}}=\right.$ $18 \pm 3 \mathrm{~nm}$ ) (Fig. S19, ESI $\dagger$ ) with no indication of aggregation, which indicates that grafting the polymer on the hydrophobic IONPs stabilizes IONPs in water at room temperature. In order to investigate the temperature responsive behavior of the P-IONp, DLS measurements were conducted at varying temperatures. At low temperatures (from 25 to $40{ }^{\circ} \mathrm{C}$ ), P-IONp revealed stable hydrodynamic diameters. However, a significant increase in the hydrodynamic size was observed at $45{ }^{\circ} \mathrm{C}$ (Fig. S20, ESI $\dagger$ ). This was attributed to the LCST behavior of the grafted polymer, which led to clustering of IONPs. ${ }^{30}$

Next, to investigate the possibility of clustering IONPs within the interior of the polymer nanogels, an aqueous suspension of P-IONp was heated at $70{ }^{\circ} \mathrm{C}$ for 30 seconds at three different concentrations (1, 2 and $\left.3 \mathrm{mg} \mathrm{mL}{ }^{-1}\right)$. After 30 seconds, diamine was added into the solution to cross-link and the solution was kept at $70{ }^{\circ} \mathrm{C}$ for 5 minutes. After cooling down to room temperature, the resulting assemblies were characterized by DLS and TEM. DLS measurements revealed that the hydrodynamic size of the resulting assemblies was larger than the hydrodynamic diameter 

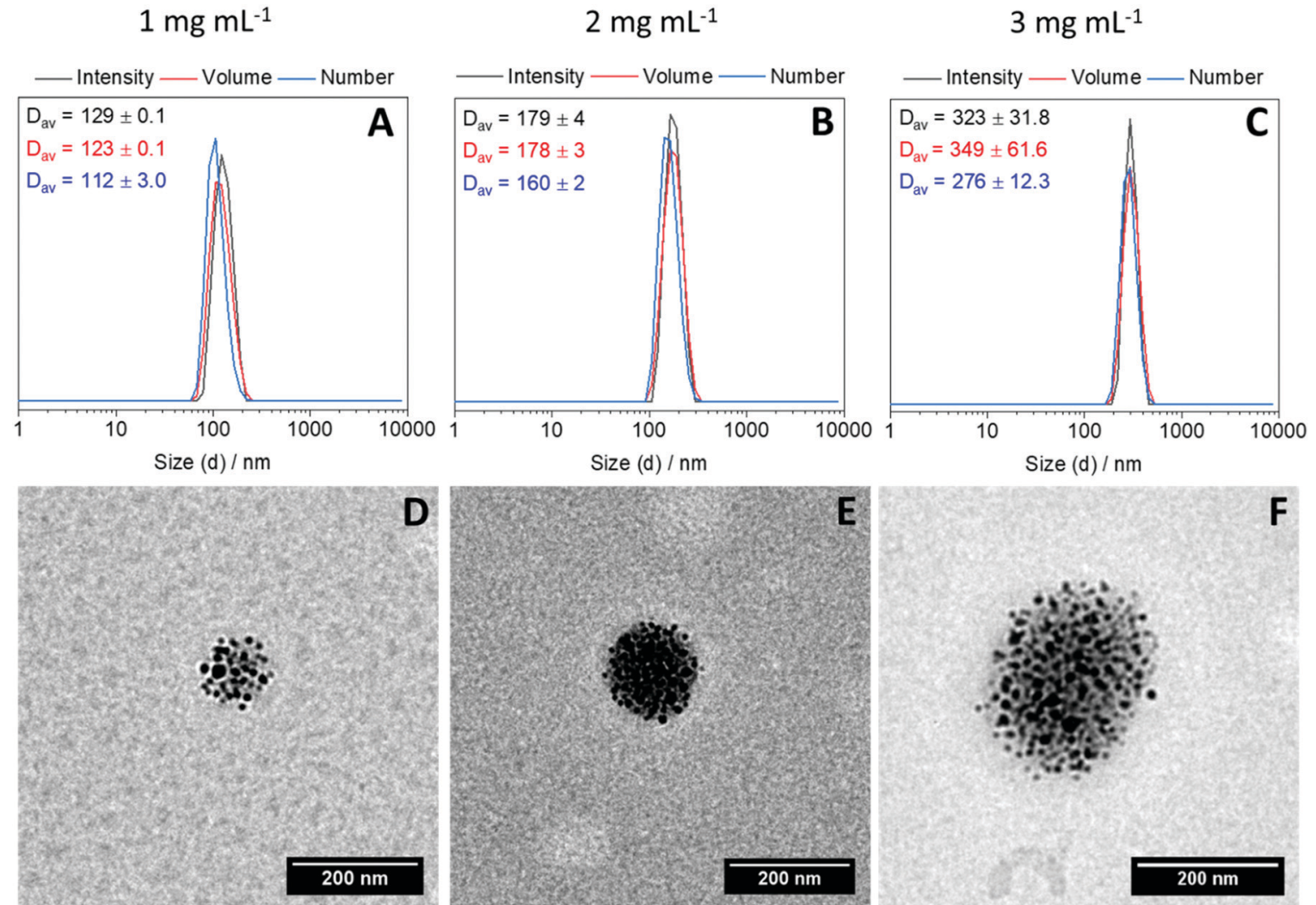

Fig. 3 DLS size distributions of the hybrid nanogels synthesized with an initial polymer grafted iron oxide nanoparticle concentration of (A) 1, (B) 2 and (C) $3 \mathrm{mg} \mathrm{mL}{ }^{-1}$, representative TEM images of the hybrid nanogels synthesized with an initial polymer grafted iron oxide nanoparticle concentration of (D) 1 , (E) 2 and (F) $3 \mathrm{mg} \mathrm{mL}^{-1}$.

of the P-IONp and the diameter of the resulting assemblies gradually increased with the increasing initial P-IONp concentration in water (Fig. 3). Zeta potential measurements revealed negative zeta potential values for all the fabricated hybrid nanogels around $-20 \mathrm{mV}$ (Fig. S21, ESI $\dagger$ ). The negative zeta potential values of the nanogels were attributed to the hydrophilic OEGMA 300 units of the polymers on the surface of the nanogels. Additionally, TEM images of the assemblies showed that IONPs were densely clustered within the well-defined spherical polymer nanogels (Fig. 3). These results indicate that size of the hybrid nanogels could be tuned simply by varying the initial concentration of the polymer grafted IONPs in water. The formation of the hybrid nanogels was attributed to the formation of larger nanosized aggregates as a result of collapse and aggregation of grafted polymer above its LCST and the subsequent in situ intra-/intermolecular diamine dichloromaleimide crosslinking. In agreement with this, the synthesized hybrid nanogels emit light with $\lambda_{\max }=$ $532 \mathrm{~nm}$ when excited at $387 \mathrm{~nm}$ (Fig. S22, ESI $\dagger$ ).

In summary, a novel dichloromaleimide functional monomer was synthesized and copolymerized with $\mathrm{OEGMA}_{300}$ via RAFT polymerization. OEGMA based dichloromaleimide bearing statistical copolymer yielded well-defined fluorescent nanogels in water when the copolymer was cross-linked using an aminedichloromaleimide reaction above the LCST of the copolymer. This copolymer could be tethered to IONPs and stabilize them in water. The LCST behavior of the grafted polymer provided a facile method for the clustering of IONPs in fluorescent polymer nanogels by heating the polymer tethered IONPs above the LCST of the grafted polymer followed by amine-dichloromaleimide cross-linking. The size of the hybrid nanogels could be tuned by simply varying the initial concentration of the polymer grafted IONPs in water. Consequently, the reported novel approach for the size-controlled synthesis of fluorescent hybrid nanogels may facilitate new opportunities for drug delivery, magnetic resonance imaging, and hyperthermia therapy.

\section{Conflicts of interest}

The authors declare no conflict of interest.

\section{References}

1 Z. Shen, T. Chen, X. Ma, W. Ren, Z. Zhou, G. Zhu, A. Zhang, Y. Liu, J. Song, Z. Li, H. Ruan, W. Fan, L. Lin, J. Munasinghe, $\mathrm{X}$. Chen and A. Wu, Multifunctional Theranostic Nanoparticles Based on Exceedingly Small Magnetic Iron Oxide Nanoparticles for T1-Weighted Magnetic Resonance Imaging and Chemotherapy, ACS Nano, 2017, 11, 10992-11004.

2 H. S. Jung, J. Han, J.-H. Lee, J. H. Lee, J.-M. Choi, H.-S. Kweon, J. H. Han, J.-H. Kim, K. M. Byun, J. H. Jung, C. Kang and J. S. Kim, Enhanced NIR Radiation-Triggered Hyperthermia by Mitochondrial Targeting, J. Am. Chem. Soc., 2015, 137, 3017-3023.

3 D. Ling, W. Park, S.-J. Park, Y. Lu, K. S. Kim, M. J. Hackett, B. H. Kim, H. Yim, Y. S. Jeon, K. Na and T. Hyeon, 
Multifunctional Tumor pH-Sensitive Self-Assembled Nanoparticles for Bimodal Imaging and Treatment of Resistant Heterogeneous Tumors, J. Am. Chem. Soc., 2014, 136, 5647-5655.

4 D. Kim, H. J. Kwon, K. Shin, J. Kim, R.-E. Yoo, S. H. Choi, M. Soh, T. Kang, S. I. Han and T. Hyeon, Multiplexible Wash-Free Immunoassay Using Colloidal Assemblies of Magnetic and Photoluminescent Nanoparticles, ACS Nano, 2017, 11, 8448-8455.

5 H. Xu, Z. P. Aguilar, L. Yang, M. Kuang, H. Duan, Y. Xiong, H. Wei and A. Wang, Antibody conjugated magnetic iron oxide nanoparticles for cancer cell separation in fresh whole blood, Biomaterials, 2011, 32, 9758-9765.

6 N. Bao and A. Gupta, Self-assembly of superparamagnetic nanoparticles, J. Mater. Res., 2011, 26, 111-121.

7 P. Qiu, C. Jensen, N. Charity, R. Towner and C. Mao, Oil Phase Evaporation-Induced Self-Assembly of Hydrophobic Nanoparticles into Spherical Clusters with Controlled Surface Chemistry in an Oil-in-Water Dispersion and Comparison of Behaviors of Individual and Clustered Iron Oxide Nanoparticles, J. Am. Chem. Soc., 2010, 132, 17724-17732.

8 R. J. Hickey, J. Koski, X. Meng, R. A. Riggleman, P. Zhang and S.-J. Park, Size-Controlled Self-Assembly of Superparamagnetic Polymersomes, ACS Nano, 2014, 8, 495-502.

9 R. J. Hickey, A. S. Haynes, J. M. Kikkawa and S.-J. Park, Controlling the Self-Assembly Structure of Magnetic Nanoparticles and Amphiphilic Block-Copolymers: From Micelles to Vesicles, J. Am. Chem. Soc., 2011, 133, 1517-1525.

10 X. Huang, J. Hu, Y. Li, F. Xin, R. Qiao and T. P. Davis, Engineering Organic/Inorganic Nanohybrids through RAFT Polymerization for Biomedical Applications, Biomacromolecules, 2019, 20, 4243-4257.

11 C. Sanson, O. Diou, J. Thévenot, E. Ibarboure, A. Soum, A. Brûlet, S. Miraux, E. Thiaudière, S. Tan, A. Brisson, V. Dupuis, O. Sandre and S. Lecommandoux, Doxorubicin Loaded Magnetic Polymersomes: Theranostic Nanocarriers for MR Imaging and Magneto-Chemotherapy, ACS Nano, 2011, 5, 1122-1140.

12 S. Lecommandoux, O. Sandre, F. Chécot, J. RodriguezHernandez and R. Perzynski, Magnetic Nanocomposite Micelles and Vesicles, Adv. Mater., 2005, 17, 712-718.

13 A. Hannecart, D. Stanicki, L. Vander Elst, R. N. Muller, A. Brûlet, O. Sandre, C. Schatz, S. Lecommandoux and S. Laurent, Embedding of superparamagnetic iron oxide nanoparticles into membranes of well-defined poly(ethylene oxide)-block-poly( $\varepsilon$-caprolactone) nanoscale magnetovesicles as ultrasensitive MRI probes of membrane bio-degradation, J. Mater. Chem. B, 2019, 7, 4692-4705.

14 J. Xie, G. Liu, H. S. Eden, H. Ai and X. Chen, SurfaceEngineered Magnetic Nanoparticle Platforms for Cancer Imaging and Therapy, Acc. Chem. Res., 2011, 44, 883-892.

15 B.-S. Kim, J.-M. Qiu, J.-P. Wang and T. A. Taton, Magnetomicelles: Composite Nanostructures from Magnetic Nanoparticles and Cross-Linked Amphiphilic Block Copolymers, Nano Lett., 2005, 5, 1987-1991.

16 K. Yang, Y. Liu, Y. Liu, Q. Zhang, C. Kong, C. Yi, Z. Zhou, Z. Wang, G. Zhang, Y. Zhang, N. M. Khashab, X. Chen and Z. Nie, Cooperative Assembly of Magneto-Nanovesicles with
Tunable Wall Thickness and Permeability for MRI-Guided Drug Delivery, J. Am. Chem. Soc., 2018, 140, 4666-4677.

17 J.-H. Ryu, S. Jiwpanich, R. Chacko, S. Bickerton and S. Thayumanavan, Surface-Functionalizable Polymer Nanogels with Facile Hydrophobic Guest Encapsulation Capabilities, J. Am. Chem. Soc., 2010, 132, 8246-8247.

18 J.-H. Ryu, R. T. Chacko, S. Jiwpanich, S. Bickerton, R. P. Babu and S. Thayumanavan, Self-Cross-Linked Polymer Nanogels: A Versatile Nanoscopic Drug Delivery Platform, J. Am. Chem. Soc., 2010, 132, 17227-17235.

19 B. Aktan, L. Chambre, R. Sanyal and A. Sanyal, "Clickable" Nanogels via Thermally Driven Self-Assembly of Polymers: Facile Access to Targeted Imaging Platforms using ThiolMaleimide Conjugation, Biomacromolecules, 2017, 18, 490-497.

20 N. Badi, Non-linear PEG-based thermoresponsive polymer systems, Prog. Polym. Sci., 2017, 66, 54-79.

21 C. Englert, J. C. Brendel, T. C. Majdanski, T. Yildirim, S. Schubert, M. Gottschaldt, N. Windhab and U. S. Schubert, Pharmapolymers in the 21st century: Synthetic polymers in drug delivery applications, Prog. Polym. Sci., 2018, 87, 107-164.

22 R. Qiao, L. Esser, C. Fu, C. Zhang, J. Hu, P. Ramírez-arcía, Y. Li, J. F. Quinn, M. R. Whittaker, A. K. Whittaker and T. P. Davis, Bioconjugation and Fluorescence Labeling of Iron Oxide Nanoparticles Grafted with BromomaleimideTerminal Polymers, Biomacromolecules, 2018, 19, 4423-4429.

23 M. P. Robin, P. Wilson, A. B. Mabire, J. K. Kiviaho, J. E. Raymond, D. M. Haddleton and R. K. O'Reilly, ConjugationInduced Fluorescent Labeling of Proteins and Polymers Using Dithiomaleimides, J. Am. Chem. Soc., 2013, 135, 2875-2878.

24 Y. Xie, J. T. Husband, M. Torrent-Sucarrat, H. Yang, W. Liu and R. K. O'Reilly, Rational design of substituted maleimide dyes with tunable fluorescence and solvafluorochromism, Chem. Commun., 2018, 54, 3339-3342.

25 M. P. Robin, M. W. Jones, D. M. Haddleton and R. K. O'Reilly, Dibromomaleimide End Functional Polymers by RAFT Polymerization Without the Need of Protecting Groups, ACS Macro Lett., 2012, 1, 222-226.

26 M. W. Jones, R. A. Strickland, F. F. Schumacher, S. Caddick, J. R. Baker, M. I. Gibson and D. M. Haddleton, Polymeric Dibromomaleimides As Extremely Efficient Disulfide Bridging Bioconjugation and Pegylation Agents, J. Am. Chem. Soc., 2012, 134, 1847-1852.

27 C. Xu, K. Xu, H. Gu, R. Zheng, H. Liu, X. Zhang, Z. Guo and B. Xu, Dopamine as A Robust Anchor to Immobilize Functional Molecules on the Iron Oxide Shell of Magnetic Nanoparticles, J. Am. Chem. Soc., 2004, 126, 9938-9939.

28 S. Imai, Y. Hirai, C. Nagao, M. Sawamoto and T. Terashima, Programmed Self-Assembly Systems of Amphiphilic Random Copolymers into Size-Controlled and Thermoresponsive Micelles in Water, Macromolecules, 2018, 51, 398-409.

29 J. Park, K. An, Y. Hwang, J.-G. Park, H.-J. Noh, J.-Y. Kim, J.-H. Park, N.-M. Hwang and T. Hyeon, Ultra-large-scale syntheses of monodisperse nanocrystals, Nat. Mater., 2004, 3, 891-895.

30 S. Kurzhals, R. Zirbs and E. Reimhult, Synthesis and MagnetoThermal Actuation of Iron Oxide Core-PNIPAM Shell Nanoparticles, ACS Appl. Mater. Interfaces, 2015, 7, 19342-19352. 